\title{
Resíduo de madeira de eucalipto e adesivo tânico em painéis aglomerados
}

\author{
Daniela Minini, Fabricio Gomes Gonçalves*, Pedro Gutemberg de Alcântara Segundinho, \\ Mayária Josiânia K. F. Sampaio Felberg, Vinícius Peixoto Tinti
}

Universidade Federal do Espírito Santo, Centro de Ciências Agrárias e Engenharias, Departamento de Ciências Florestais e da Madeira.

\begin{abstract}
RESUMO Esta pesquisa teve como objetivo avaliar a utilização de serragem proveniente do seccionamento transversal de toras de eucalipto de uma destopadeira para produção de painéis aglomerados. Foram produzidos painéis com densidade nominal de 0,60 g. $\mathrm{cm}^{-3}$ e partículas que passassem pela peneira de $2 \mathrm{~mm}$ e ficassem retidas na peneira de $1,5 \mathrm{~mm}$. Foi utilizado o adesivo ureia formaldeído puro e em associação com uma solução de tanino comercial de Acacia mearnsii (1:1) com água destilada. Aplicou-se às partículas adesivo nas proporções de $0 \%, 2 \%, 5 \%, 10 \%$ e $15 \%$ em substituição ao adesivo químico comercial. As seguintes propriedade físicas e mecânicas dos painéis foram analisadas: absorção de água após 2 e 24 horas de imersão, densidade aparente, inchamento em espessura após 2 e 24 horas de imersão, ligação interna, arranchamento de parafuso (topo e face) e flexão estática. Os resultados indicaram que para os painéis confeccionados, o tipo de partícula, conjuntamente com os adesivos modificados pelo tanino, apresenta potencialidade limitada de uso, indicando qualidade inferior aos do adesivo comercial ureia formaldeído.
\end{abstract}

Palavras-chave: resíduo de serraria; tanino; adesivo natural; polifenol.

\section{Waste of eucalyptus wood and tannic adhesive in particleboards}

\begin{abstract}
The objective of the research was study the sawdust eucalypt wood obtained from cross-cut in the production particleboards. These were made with nominal density of $0.60 \mathrm{~g} \mathrm{~cm}^{-3}$ and particles that passed through the $2.0 \mathrm{~mm}$ sieve and was stayed in the $1.5 \mathrm{~mm}$ sieve. Utilized the adhesive commercial urea formaldehyde pure and homogenized with tannin solution Acacia mearnsii (1:1) with distilled water. Has been applied at the particles, adhesive on the proportions of $0 \%, 2 \%, 5 \%$, $10 \%$ and $15 \%$ in replacing to chemical adhesive commercial. The properties evaluated were: water absorption and thickness swelling after 2 and 24 hours, bulk density, internal bond, screw withdrawal (top and side), and static bending. The results indicated that for particleboards, particle type, together with the adhesives modified by the tannin, presented limited use potential, indicating a lower quality to the commercial adhesive urea formaldehyde.
\end{abstract}

Keywords: wood waste; tannin; natural adhesive; polyphenol.

\section{Introdução}

Os painéis de madeira aglomerada, comercialmente denominado de MDP (Medium Density Particleboard), são produzidos com partículas de madeira, com a incorporação de um adesivo sintético, e consolidado por meio de aplicação de calor e pressão em uma prensa aquecida. Contudo, outros materiais lignocelulósicos podem ser utilizados na fabricação de aglomerados (GHALEHNO; NAZERIASN, 2012; MALONEY, 1993; PEDRAZZI et al., 2006). No campo ambiental, o reaproveitamento de resíduos madeireiros pode propiciar sustentabilidade, minimizar problemas de poluição ambiental e auxiliar no atendimento da demanda das indústrias de painéis aglomerados (NEGRÃO et al., 2014), além de maximizar o uso da matéria-prima.

O processo de fabricação de painéis aglomerados passou por inúmeras adequações ao longo do tempo, principalmente, quanto às limitações técnicas, como absorção de água, 
inchamento em espessura, usinabilidade das bordas e problemas quanto à fixação de parafusos (MELO; DEL MENEZZI, 2010). Novas tecnologias no setor de painéis, como o uso de parafina, controle do gradiente de massa específica, sistema de fixação de parafusos mais eficientes, bem como novas propostas de adesivos e misturas de espécies florestais com variadas densidades foram sendo incorporadas (SANCHES et al.; 2016), o que permitiu minimizar os problemas de qualidade.

$\mathrm{Na}$ manufatura de produtos reconstituídos de madeira, o adesivo é um componente fundamental, por ser responsável pela ligação entre a madeira e a transferência de tensões geradas na linha de cola durante a sua utilização, além de participar significativamente na composição de custos de produção (IWAKIRI et al., 2000). Especificamente, em painéis aglomerados, o adesivo sintético tipicamente utilizado é a ureia formaldeído (UF) (STARK et al., 2010).

A utilização de tanino, produto renovável, vem se destacando com o passar os anos, onde a geração de conhecimentos científicos sobre seu uso e aplicação como adesivo é estudado em várias academias (ANTWI-BOASIAKO; ANIMAPAUH (2012), CARNEIRO et al. (2004), CARNEIRO et al. (2009), CARVALHO et al. (2014), DEL CARVALHO (2010), ELBADAWI et al. (2015), GONÇALVES et al. (2008)). O tanino extraído de acácia negra (Acacia mearnsii) é o único explorado comercialmente por empresas localizadas no estado do Rio Grande do Sul, e que o produzem para diversas aplicações, dentre elas, o adesivo.

Com base no exposto, propõe-se a hipótese de que a utilização de tanino adicionado à ureia formaldeído em partículas de eucalipto, não influenciará de forma negativa a nas propriedades físico e mecânicas dos painéis produzidos. Nesse sentido, objetivou-se avaliar a qualidade de painéis aglomerados, confeccionados com partículas de madeira de eucalipto proveniente de destopadeira (serra circular), com adição de uma solução de tanino como elemento modificador em resina sintética ureia formaldeído.

\section{Material e Métodos}

\section{Coleta do material de pesquisa}

As partículas foram obtidas em uma serraria localizada na região do Caparaó, sul do Estado do Espírito Santo, sendo exclusivamente de madeira de eucalipto, e proveniente de serra destopadeira. $\mathrm{O}$ material foi seco ao ar, peneirado em peneira de $4 \mathrm{~mm}$; na sequência, em peneira de $2 \mathrm{~mm}$, sendo utilizadas na fabricação dos painéis aquelas partículas que passaram nesta última e ficaram retidas na peneira de malha de $1,5 \mathrm{~mm}$. A matéria-prima utilizada continha casca em sua composição, portanto não sendo possível sua quantificação e ou exclusão. Também foram coletadas amostras aleatórias de cascas e madeira para análises químicas.

Em seguida as partículas foram secas em estufa de circulação e renovação forçada de ar com temperatura de $80^{\circ} \mathrm{C}$ até umidade de 5\% (base seca) e armazenadas em sacos plásticos hermeticamente fechados.

\section{Análise física e química dos resíduos e dos}

\section{adesivos}

$\mathrm{Na}$ matéria-prima (casca, madeira, partículas) foi analisado o pH e tamponamento (Gonçalves; Lelis, 2012), o teor de cinzas conforme estabelecido pela Associação Brasileira Técnica de Celulose e Papel - ABTCP M 11/77 (1974), os extrativos (TAPPI, 1998). A densidade básica foi quantificada de acordo com a Norma Brasileira Regulamentadora - NBR 11941, da Associação Brasileira de Normas Técnicas - ABNT (2003), sendo as análises realizadas em peças de madeira e cascas coletadas aleatoriamente na pilha de resíduo próximo a destopadeira. 
$\mathrm{O}$ adesivo químico puro e modificado com tanino foi caracterizado quanto às suas propriedades tecnológicas $(\mathrm{pH}$, viscosidade e teor de sólidos). Foi utilizada a resina comercial ureia formaldeído, obtida em indústria química, alterada com uma solução de tanino comercial de Acacia mearnsii a 50\% com água destilada, sendo homogeneizada até o ponto em que não se observou mais a presença de grumos na solução. Não foi realizada alteração do $\mathrm{pH}$ nos adesivos.

\section{Formação do colchão e parâmetros de}

\section{produção}

Após a obtenção da massa das partículas e quantificação do adesivo, de acordo com cada tratamento estabelecido, foi adicionada a resina já com o catalizador (sulfato de amônio a $24 \%$ em proporção de $2 \%$ sobre a massa total de sólidos de cada adesivo) por meio de aspersão em encoladeira rotativa.

Para a formação do colchão, as partículas já encoladas foram acomodadas em uma caixa formadora com dimensões de 42,5 x 42,5 cm e distribuídas uniformemente de forma manual. Antes da prensagem, o colchão de partículas foi disposto entre duas chapas metálicas de alumínio, com dois espaçadores de 1,25 cm de espessura dispostos entre as duas chapas.

Os parâmetros de fabricação foram: temperatura da prensa de $140^{\circ} \mathrm{C}$ por 8 minutos e pressão de $3,92 \mathrm{MPa}$, utilizando-se um teor de $8 \%$ de adesivo sobre a massa seca, em todos os tratamentos. Após a prensagem, os painéis foram mantidos em ambiente climatizado com umidade relativa de $65 \pm 5 \%$ e temperatura de $20 \pm 5^{\circ} \mathrm{C}$. Posteriormente foram esquadrejados e obtidos os corpos de prova para realização dos ensaios físicos e mecânicos.

\section{Ensaios físicos e mecânicos nos painéis}

Foram realizados os ensaios de densidade aparente, inchamento em espessura (após 2 e 24 horas de imersão em água), absorção de água (após 2 e 24 horas de imersão em água) - ambos a temperatura ambiente, ligação interna, flexão estática (módulos de ruptura e de elasticidade), e arrancamento de parafuso (topo e superfície) conforme estabelecido pela Norma Brasileira Regulamentadora - NBR 14.810-3 (ABNT, 2002). Confeccionados os corpos de prova, os mesmos permaneceram em ambiente climatizado por um período de 15 dias (umidade relativa de $65 \pm 5 \%$ e temperatura de $\left.20 \pm 5^{\circ} \mathrm{C}\right)$

Os resultados dos testes foram comparados com os valores estabelecidos pela norma Commercial Standard CS 23666 (1968) e da Associação Brasileira de Normas Técnicas ABNT (2006).

\section{Tratamentos estudados e delineamento}

\section{estatístico}

$\mathrm{Na}$ análise variância, com base no delineamento experimental inteiramente casualisado, adotou-se um nível de significância de 5\%. Foram confeccionados três painéis (repetições) para cada tratamento, totalizando 18 painéis. Havendo diferença estatística entre os tratamentos (Tabela 1), foi procedido o teste de Tukey a 5\% de significância. Anteriormente, os dados foram submetidos à análise de normalidade (Kolmogorov-Smirnov: $\mathrm{p}>0,05)$ e teste de homocedastia (Bartlett: $\mathrm{p}>0,01)$. Os dados não foram transformados.

Tabela 1. Composição dos tratamentos utilizados.

Table 1. Composition of treatments.

\begin{tabular}{cccc}
\hline $\begin{array}{c}\text { Trata- } \\
\text { mento }\end{array}$ & $\begin{array}{c}\text { Ureia for- } \\
\text { maldeído } \\
(\%)\end{array}$ & $\begin{array}{c}\text { Tanino for- } \\
\text { maldeído* } \\
(\%)\end{array}$ & Sigla \\
\hline T1 & 100 & - & UF \\
T2 & - & 100 & TF \\
T3 & 98 & 2 & $98: 2$ TUF \\
T4 & 95 & 5 & $95: 5$ TUF \\
T5 & 90 & 10 & $90: 10$ TUF \\
T6 & 85 & 15 & $85: 15$ TUF \\
\hline
\end{tabular}

* Solução de tanino comercial de Acacia mearnsii com 50\% em água destilada. 


\section{Resultados e Discussão}

\section{Características químicas das partículas}

As partículas foram originadas de madeira com densidade básica média igual a $0,56 \mathrm{~g} \cdot \mathrm{cm}^{-3}\left( \pm 0,04 \mathrm{~g} \cdot \mathrm{cm}^{-3}\right)$, próximo aos valores encontrados por Boa et al. (2014), que ao caracterizarem resíduos madeireiros de diferentes espécies de eucalipto encontraram densidade no intervalo de 0,50 e 0,81 g.cm ${ }^{-3}$. O conhecimento da densidade da madeira é importante pois permite obter a taxa de compressão das partículas na formação de painéis aglomerados (MOSLEMI, 1974; MALONEY, 1993).

Os valores de $\mathrm{pH}$ oscilaram entre 4,2 a 4,96 e para o tamponamento das partículas utilizadas de 0,01819 a 0,0903 nmmolNaOH (Tabela 2).

$\mathrm{O}$ resíduo utilizado apresentou valores de $\mathrm{pH}$ tendendo para ácido, como era de se esperar (MALONEY, 1993; PIZZI; MITTAL, 1994), uma vez que a maioria das espécies florestais apresentam pH ligeiramente ácido (IWAKIRI, 2005). Valores de $\mathrm{pH}$ ligeiramente mais elevado são encontrados na madeira em relação à casca, sendo uma característica inerente às espécies florestais (ROWELL et al., 2005) devido sobretudo a maior atividade cambial (presente nas células divisoras localizadas entre a casca interna e a madeira) e da presença de extrativos. No entanto, neste trabalho, os resultados mostraram valores ligeiramente superiores para a casca (Tabelas 2 e 3 ) com extração em água fria. Isso se deve sobretudo a origem da mesma, idade e manejo adotado, uma vez que a fonte da matéria-prima é diversificada. A presença de casca nas partículas na formação de um painel aglomerado feito com madeira de Eucalyptus cinerea proporcionou um ligeiro aumento do pH nas mesmas (PAN et al., 2007), no entanto, as propriedades tecnológicas não foram comprometidas.

Trabalhando com a madeira de Acacia mangium na fabricação de painéis aglomerados homogêneos, Gonçalves; Lelis (2012), encontraram valores de $\mathrm{pH}$ que variaram de 5,45 a 5,90 e valores parecidos de capacidade tampão, sendo de 0,05 a 0,104 mmolNaOH. A observação do valor do $\mathrm{pH}$ na madeira possui uma severa importância, principalmente no que se refere a cura do adesivo, sobretudos naquelas resinas à base de formaldeído (IRLE; BARBU, 2010). Desta forma, um adesivo pode não curar o suficientemente, na presença de uma madeira com pH muito ácido, havendo a necessidade de um pré-tratamento em suas partículas, evitando a formação de ligações fracas entre o adesivo e o aderente (IRLE; BARBU, 2010). Ainda, pode haver a ocorrência de polimerização parcial do adesivo que cura em meio ácido, tal como ureia formaldeído, o que comprometeria a integridade da ligação (FRIHART; HUNT, 2010).

Os menores valores encontrados para o tamponamento estiveram entre 0,0903 e $0,0422 \mathrm{mmolNaOH} .5 \mathrm{~g}^{-1}$, respecti-

Tabela 2. Valores médios de pH e capacidade tampão ácida para os resíduos de eucalipto.

Table 2. Average values of $\mathrm{pH}$ and acid buffering capacity for eucalypt waste.

\begin{tabular}{cccc}
\hline Modo e tempo de extração & Matéria-prima & $\mathbf{p H}$ & $\begin{array}{c}\text { Capacidade tampão ácida } \\
\left(\mathbf{m m o l N a O H} \mathbf{5} \mathbf{g}^{-1}\right)\end{array}$ \\
\hline Água fria (ambiente) - 24 horas & Casca & 4,96 & 0,0903 \\
Água quente -20 min & & 4,27 & 0,1819 \\
\hline Água fria (ambiente) - 24 horas & \multirow{2}{*}{ Partículas com casca } & 4,86 & 0,0340 \\
Água quente - 20 min & & 4,46 & 0,0695 \\
\hline Água fria (ambiente) - 24 horas & Madeira & 4,20 & 0,0422 \\
Água quente -20 min & & 4,69 & 0,0622 \\
\hline
\end{tabular}

Tabela 3. Valores médios de teor de cinzas e de extrativos para os resíduos de eucalipto. 
Table 3. Average values content of ash and extractives for eucalypt waste.

\begin{tabular}{ccc}
\hline Resíduo & Teor de Cinzas (\%) & Teor de Extrativos (\%) \\
\hline Casca & 2,11 & 11,38 \\
Partículas com casca & 0,47 & 6,40 \\
Madeira & 0,22 & 7,67 \\
\hline Média & $0,93(0,89)^{\star}$ & $8,48(2,90)$ \\
\hline
\end{tabular}

* Valor entre parênteses corresponde ao desvio padrão.

vamente para casca e para a madeira de eucalipto, após extração em água fria. Para as partículas utilizadas no processo, o maior valor de tamponamento foi 0,0695 mmolNaOH. $5 g^{-1}$ após extração em água quente. Uma vez que o pH mede a acidez da madeira e ou na casca, a capacidade tampão, de acordo com Maloney (1993) mede a resistência da madeira em alterar o $\mathrm{pH}$; isso quer dizer que altos valores de capacidade tampão necessitam de quantidades maiores de catalisadores ácidos, para que assim, o $\mathrm{pH}$ possa ser reduzido visando a cura ideal do adesivo.

O teor médio de cinzas encontrado foi de $0,93 \%$ (Tabela 3). A presença de elementos inorgânicos na casca, como destacado por Rowell et al. (2005) e Wegner et al. (1989), é maior se comparado à madeira, sendo também as condições ambientais em que a árvore se desenvolve um importante fator a ser considerado. Os teores médios de cinzas na madeira, normalmente oscilam entre $0,3 \%$ a $1,5 \%$, com destaque para o cálcio (500-1200 ppm), o potássio (200-1000 ppm) e o magnésio (100-300 ppm). Outros elementos como manganês, sódio, cloro, fósforo, alumínio e zinco estão presentes em quantidades inferiores a 100 ppm (WEGNER et al., 1989). Estes, quando presentes, em quantidades elevadas, podem comprometer os processos de adesão entre o aderente e o adesivo por interferirem no mecanismo de trocas entre as pontes de hidrogênio livres.

Os extrativos de madeira, conforme mencionado por Frihart; Hunt (2010) podem interferir com o contato direto com o adesivo, levando a um efeito de limite quimicamente fraco e à uma baixa resistência de união com o aderente. De acordo com Iwakiri (2005), valores baixos de $\mathrm{pH}$, podem contribuir para uma pré-endurecimento da ureia formaldeído, por exemplo. Assim, o pH da madeira define se o processo reativo do mecanismo da adesão pode ser acelerado ou não, dificultando a ligação entre o aderente e o adesivo. No caso específico do valor encontrado para o extrativo nas partículas com casca esperava-se um valor superior, o que não aconteceu, possivelmente a casca presente não deve ter influenciado de forma significativa a extração de compostos de caráter mais ácidos.

\section{Características químicas dos adesivos}

\section{utilizados}

Os valores encontrados para o $\mathrm{pH}$, teor de sólidos e viscosidade na solução tânica pura (Tabela 4) apesentaram pequenas variações em relação aos outros trabalhos do mesmo gênero (GONÇALVES; LELIS, 2008, GONÇALVES; LELIS, 2009, CARVALHO et al. 2014).

À medida que foi adicionado tanino ( $\mathrm{pH}$ ácido) a ureia formaldeído ( $\mathrm{pH}$ alcalino), ocorreu uma redução dos valores de $\mathrm{pH}$; isso ocorreu por ser adicionado um catalizador que libera ácido para o meio (sulfato de amônio), acelerando processo de cura das resinas (GONÇALVES; LELIS, 2008). Isto pode ter contribuído para a dificuldade de obtenção da viscosidade, comprometendo a integridade do equipamento, uma vez que o mesmo fornecia dados muito elevados, quando comparados à bibliografia. Devido a este fato os mesmos não foram apresentados. 
Tabela 4. Valores médios do teor de sólido (\%), $\mathrm{pH}$ e viscosidade para os adesivos estudados.

Table 4. Average values of solid content (\%), $\mathrm{pH}$ and viscosity for the adhesives studied.

\begin{tabular}{|c|c|c|c|}
\hline Tratamentos $^{*}$ & $\begin{array}{c}\text { Teor de sólidos } \\
(\%)\end{array}$ & $\mathrm{pH}$ & $\begin{array}{c}\text { Viscosidade } \\
\text { (MPa) }\end{array}$ \\
\hline $\mathbf{1}(100 \% \mathrm{UF})$ & 72,05 & 8,26 & 1.200 \\
\hline $2(100 \% \mathrm{TF})$ & 51,61 & 3,52 & 580 \\
\hline 3 (98\% UF+2\% TF) & 76,12 & 7,13 & nd \\
\hline $4(95 \% \mathrm{UF}+5 \% \mathrm{TF})$ & 73,21 & 5,99 & nd \\
\hline 5 (90\% UF+10\% TF) & 71,56 & 5,26 & nd \\
\hline $6(85 \% \mathrm{UF}+15 \% \mathrm{TF})$ & 67,88 & 4,94 & nd \\
\hline
\end{tabular}

* UF: Ureia formaldeído, TF: Tanino formaldeído, nd: não determinado

\section{Propriedades físicas dos painéis}

Os valores médios de densidade aparente dos painéis variaram de 0,35 a 0,51 g.cm $\mathrm{cm}^{-3}$, sendo 0,43 g.cm $\mathrm{cm}^{-3} \mathrm{o}$ valor médio encontrado entre os tratamentos (Figura 1). A densidade almejada para os painéis de $0,60 \mathrm{~g} . \mathrm{cm}^{-3}$, não foi alcançada e conforme a norma CS 236-66 (1968), os painéis são enquadrados como sendo de baixa densidade (inferior a 0,60 g.cm ${ }^{-3}$ ). Os baixos valores de densidade obtidos possibilitaram valores reduzidos da taxa de compactação em todos os tratamentos: T1: 0,79; T2: 0,83; T3: 0,70; T4: 0,74; T5: 0,79; T6: 0,79, o que segundo Maloney (1993), deveria estar mais próximo de 1,3.

Apesar da densidade ser uma variável facilmente controlável no processo, isso não ocorreu. Possivelmente por estar associada às condições de processamento anteriores à operação de prensagem como, por exemplo, da preparação e secagem da madeira, baixo teor de adesivo utilizado e arranjo das partículas no colchão (HASELEIN et al., 2002; MALONEY, 1993; MOSLEMI, 1974; PAN et al., 2007) e ainda a fatores como a origem e o tipo de partícula (sem padrão de espessura e a heterogeneidade), bem como a quantidade de casca (fator não controlável).

Os valores médios de inchamento em espessura após 2 e 24 horas de imersão, variaram de $13,22 \%$ a $38,20 \%$ e $19,11 \%$ a 41,55\%, respectivamente (Figura 2). Conforme estabelecido pela CS 236-66 (1968) o valor máximo para inchamento é de
$30 \%$, após $24 \mathrm{~h}$, visto que na pesquisa somente o T2 está acima do valor permitido. O valor médio geral encontrado foi de $24,43 \%$ e $28,43 \%$, respectivamente para IE2 e IE24.

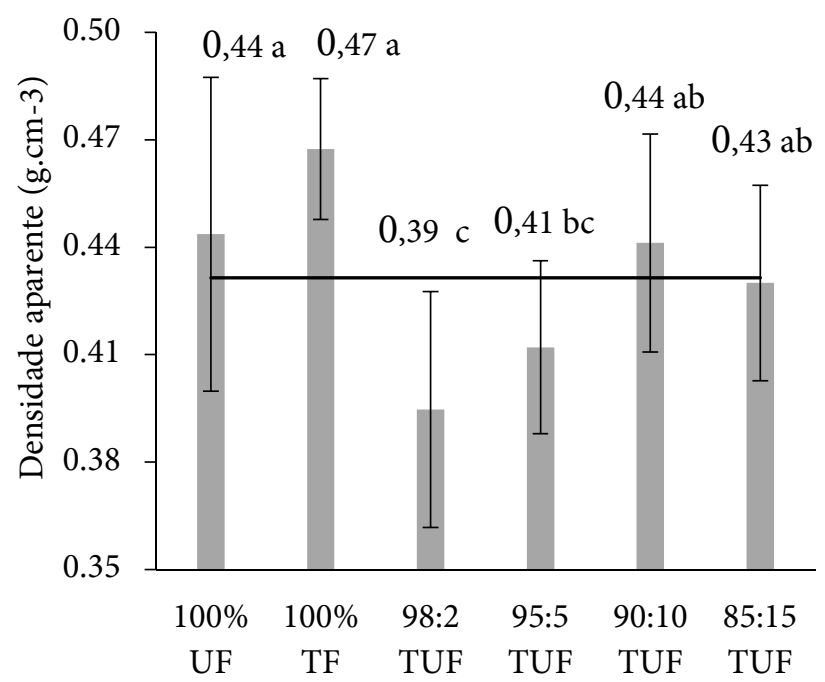

Figura 1. Densidade aparente dos painéis aglomerados avaliados. Médias seguidas pela mesma letra não diferem entre si estatisticamente (Tukey p < 0,05). Barra é o desvio padrão. Traço é a média geral.

Figure 1. Bulk density of the studied particleboards. Means followed by the same letter are not statistically different (Tukey $\mathrm{p}<0.05$ ). Bar is the standard deviation. Risk is the general average.

Para o inchamento em espessura em 2 horas de imersão, o T2 (100\% de tanino), que apresentou a maior razão de compactação $(0,83)$, obteve um aumento significativo no inchamento $(28,97 \%)$, resultado este ruim para este teste, e os tratamentos com teores de tanino não apresentaram diferenças estatísticas, estes, com razão de compactação entre 0,7 a 0,79. 
Após 24 horas de imersão, observou-se no inchamento em espessura que os tratamentos não apresentaram diferenças estatísticas entre as médias, apresentando 28,43\% como valor médio.
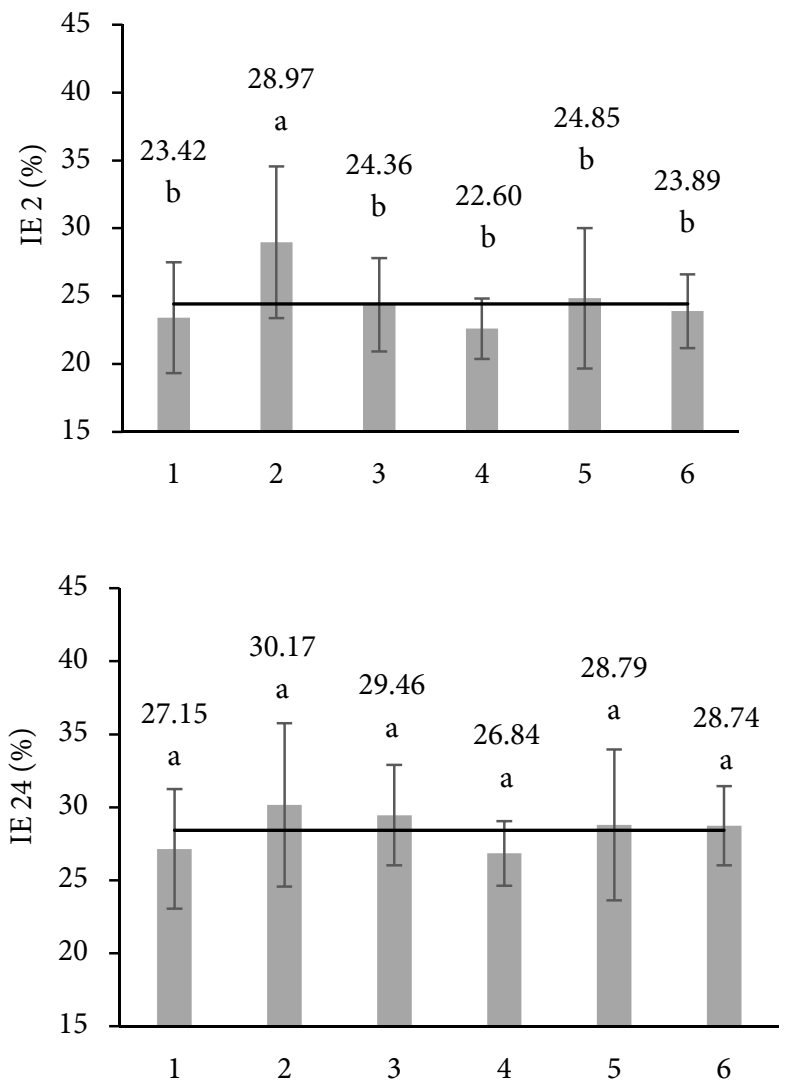

Figura 2. Valores médios de inchamento em espessura após 2 horas (IE2) e 24 horas de imersão (IE24). Médias seguidas pela mesma letra não diferem estatisticamente entre si (Tukey $<0,05)$. Barra é o desvio padrão. Traço é a média geral.

Figure 2. Average values of thickness swelling for 2 hours (IE2) and 24 hours of immersion (IE24), in percentage. Means followed by the same letter aren't statistically different (Tukey < 0.05). Bar is standard deviation. Risk is the general average.

Os valores médios de inchamento em espessura após 2 e 24 horas de imersão, variaram de $13,22 \%$ a $38,20 \%$ e $19,11 \%$ a $41,55 \%$, respectivamente (Figura 2). Conforme estabelecido pela CS 236-66 (1968) o valor máximo para inchamento é de $30 \%$, após 24 h, visto que na pesquisa somente o T2 está acima do valor permitido. O valor médio geral encontrado foi de $24,43 \%$ e $28,43 \%$, respectivamente para IE2 e IE24.
As propriedades físicas de painéis aglomerados de $E u$ calyptus grandis colados com ureia formaldeído (UF) e tanino formaldeído (TF) de acácia negra, permitiram Melo et al. (2010) concluírem que o inchamento em espessura após 2 horas utilizando a resina UF foi mais instável dimensionalmente em relação aos que utilizaram TF. Após 24 h de imersão em água, os painéis confeccionados com UF apresentaram inchamento em espessura superior aos painéis confeccionados com TF.

Os valores médios para o ensaio de absorção de água após 2 e 24 horas de imersão, oscilaram de $126,33 \%$ a $210,55 \%$ e 113,16 a 237,29\%, respectivamente (Figura 3). O valor médio geral encontrado foi de $172,75 \%$ e $174,54 \%$, respectivamente para AA2 e AA24.
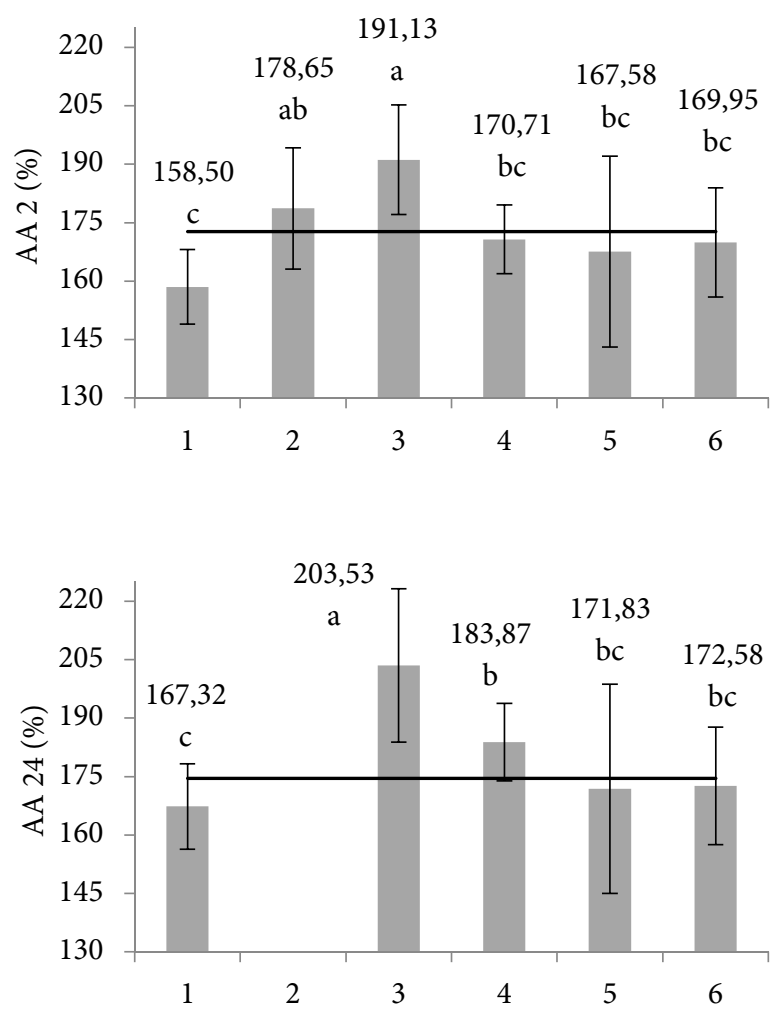

Figura 3. Valores médios de absorção de água após 2 horas (AA2) e 24 horas de imersão (AA24). Médias seguidas pela mesma letra não diferem estatisticamente (Tukey < 0,05). Barra é o desvio padrão. Traço é a média geral.

Figure 3. Average values of water absorption in (\%) after immersion for 2 hours (IE2) and 24 hours of immersion (IE24). Means followed by the same letter aren't statistically different 
(Tukey $<0.05)$. Bar is standard deviation. Risk is the general average.

De acordo com os resultados de Elbadawi et al. (2015), a adição de tanino ao adesivo químico não melhorou as propriedades físicas dos painéis (inchamento em espessura e absorção de água); quando comparado com a presente pesquisa, algumas adições de tanino permitiram melhorias no inchamento e absorção de água após 24 horas (5\% e 10\% de tanino).

O adesivo com 100\% de ureia formaldeído (razão de compactação de 0,79 ), obteve o menor valor relativo (AA2) em relação àqueles tratamentos com substituição da ureia pelo tanino, porém não diferindo entre elas. O que é interessante, pois absorção de água foi menor nestes tratamentos, por outro lado, o incremento de apenas $2 \%$ de tanino permitiu maiores valores de absorção de água após 2 horas.

Após 24 horas de absorção, boa parte dos corpos de prova do tratamento $100 \% \mathrm{TF}$, foram desintegrados devido a fraca adesão e a liberação das tensões, ficando a média comprometida. Neste trabalho ficou evidente que o adesivo com $100 \%$ de tanino não suporta longos período de contato com água, devido à sua molécula ser hidrofílica. De certa forma, contradiz algumas informações na literatura, que menciona que adesivos a base de tanino possui resistência a umidade (PIZZI; MITTAL, 2003).

A substituição parcial de tanino formaldeído (TF) à ureia formaldeído (UF) em painéis aglomerados de Eucalyptus grandis, após 2 horas de imersão, de acordo com Melo et al. (2010), não influenciou na absorção de água. Após 24 horas de imersão, os painéis não apresentaram variações significativas de absorção de água, destacando que naqueles painéis onde houveram modificação com tanino formaldeído ocorreram menores valores de absorção.
Maiores valores de absorção de água após 2 horas de imersão em água foram observados em painéis encolado com ureia e tanino formaldeído (OKINO et al., 1997), sendo condizente com o mencionado por Melo et al. (2010) por ser reconhecidamente a UF um adesivo de considerável resistência à umidade quando submetida a um rápido contato. Este comportamento diferenciado entre o inchamento e absorção se deve às diferentes razões de compactação dos painéis (DACOSTA et al., 2005).

Espera-se que em painéis confeccionados com ureia formaldeído ocorra maior estabilidade dimensional em relação àqueles em que se utilizou tanino formaldeído (CARNEIRO et al., 2004). Isso poderia indicar que, embora adesivos com tanino possam apresentar considerável resistência à umidade (MELO et al., 2010), a qualidade desses adesivos e de sua adesão depende muito de sua formulação, do material de origem e de tratamentos realizados para melhoria de sua eficiência.

Naqueles tratamentos em que se utilizaram o tanino como adesivo puro ou com adição de 2, 5, 10 e 15\% de tanino à UF (T3, T4, T5, T6, respectivamente), foram encontrados valores decrescentes de absorção de água, indicando que o aumento da proporção a adição foi positiva até o máximo de $10 \%$. O adesivo ureia formaldeído (100\% UF) absorveu menor quantidade de água em relação aos demais, sendo portando o valor mais satisfatório para o ensaio.

\section{Propriedades mecânicas dos painéis}

Os valores médios encontrados para MOR variaram de 0,588 a 2,842 $\mathrm{MPa}$, com média geral igual a 1,48 MPa. A utilização de 2\% de tanino à ureia formaldeído (98:2) apresentou resultado inferior (menor média encontrada) para com os demais, porém, não diferindo-se estatisticamente daqueles em que se utilizou $100 \%$ de ureia formaldeído, 5\% e $10 \%$ de tanino formaldeído (Figura 4). 

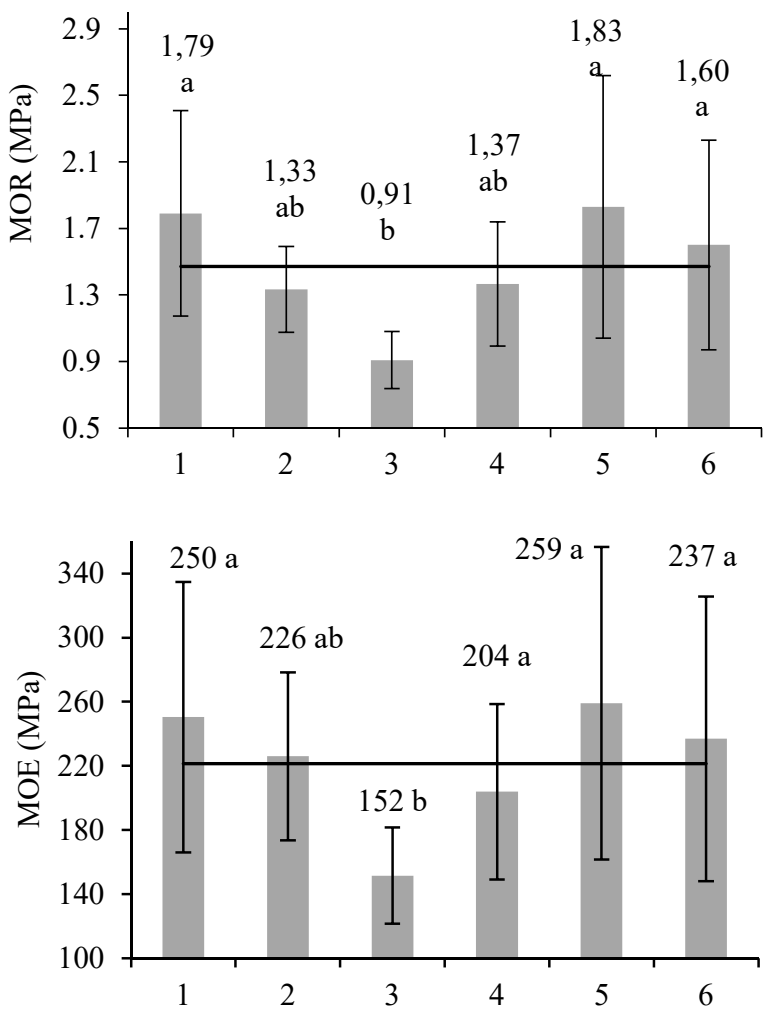

Figura 4. Valores médios observados para os ensaios mecânicos: Módulos de ruptura (MOR) e elasticidade (MOE) em flexão estática. Médias seguidas pela mesma letra não diferem estatisticamente entre si (Tukey <0,05). Barra é o desvio padrão. Traço é a média geral.

Figure 4. Average values of the modulus of rupture (MOR) and elasticity (MOE) in static bending. Means followed by the same letter aren't statistically different (Tukey <0.05). Bar is standard deviation. Risk is the general average.

Os valores encontrados para MOE variaram de 78,30 a $388,08 \mathrm{MPa}$ (média geral igual a 221,05 MPa), com o comportamento estatístico similar ao MOR, onde os tratamentos T1, T2, T4, T5 e T6 não diferiram entre si. O tratamento T3 (2\% de tanino formaldeído) apresentou o menor valor absoluto (Figura 4). Os valores das médias da presente pesquisa para MOE apresentam-se muito abaixo se comparados a trabalhos já realizados com as características similares (CUNHA et al., 2014; HASELEIN et al., 2002; IWAKIRI et al., 2000; MELO DEL MENEZZI, 2010). Em relação a ABNT (2006), a mesma não estabelece valores de referência para MOE.
Os valores encontrados para MOR na presente pesquisa situam-se abaixo daqueles encontrados por outros trabalhos de características próximas a este (IWAKIRI et al., 2000; MELO, DEL MENEZZI, 2010), não atendendo também ao especificado pela NBR 14.810-3 (ABNT, 2006) que estabelece $16 \mathrm{MPa}$ como limite inferior.

Diante das médias obtidas em cada tratamento, foi observado que a adição de tanino ao adesivo ureia formaldeído não promoveu melhorias expressivas nas propriedades estudadas, visto que a maior média encontrada foi no tratamento T1 (100\% de UF). A adição parcial de tanino ao adesivo UF não promoveu aumento da resistência em painéis aglomerados para nenhum dos ensaios realizados por Gonçalves et al. (2008).

Os resultados para o ensaio de ligação interna foram de 0,82 a 6,28 MPa, com média geral igual a 1,67 MPa. Todos tratamentos estudados apresentaram valores médios acima do mínimo estabelecido pela norma CS 263-66 (1968) que é de 0,14 Mpa, para painéis inferiores a 0,60 g.cm ${ }^{-3}$ (Figura 5).

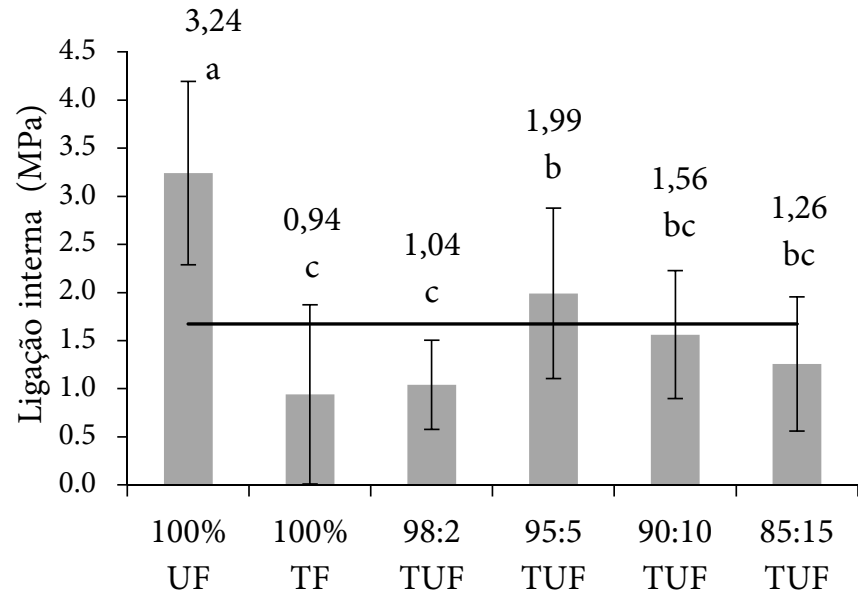

Figura 5. Valores médios do ensaio de resistência à ligação interna (LI) dos painéis. Médias seguidas pela mesma letra não diferem estatisticamente entre si (Tukey <0,05). Barra é o desvio padrão. Traço é a média geral.

Figure 5. Average values of internal bond. Means followed by the same letter aren't statistically different (Tukey <0.05). Bar is standard deviation. Risk is the general average. 
O acréscimo de tanino na resina ureia formaldeído teve influência sobre a ligação interna, uma vez que os dados apresentaram uma tendência de queda à medida que se aumentou a proporção de tanino. Em painéis aglomerados com madeira de Eucalyptus benthamii, Eucalyptus dunnii e Eucalyptus grandis, Cunha et al. (2014), encontraram resultados para a ligação interna variando entre 0,71 e 1,18 MPa, inferiores ao deste estudo. Possivelmente o mecanismo de adesão proporcionado pelo agente tânico associado à presença de casca contribuiu para este comportamento.

Os valores encontrados para o ensaio de arrancamento de parafuso na superfície e no topo dos painéis aglomerados variaram de 0,30 a 4,92 Mpa e 1,02 a 4,65 $\mathrm{MPa}$, respectivamente, sendo os valores médios representados graficamente na Tabela 5 .

O arrancamento de parafuso na superfície e no topo, em todos os tratamentos, não atingiu o mínimo estipulado pela NBR 14810-2 (ABNT, 2006), que é de 7,99 e 10,19 MPa, respectivamente. Apesar dos baixos valores encontrados, isso não impede o uso dos painéis em situações em que esta propriedade é pouco utilizada, como por exemplo em paredes em isolamento acústico. Possivelmente a dimensão reduzida das partículas contribuiu para os baixos valores encontrados. A adição de tanino ao adesivo químico permitiu pequenas elevações de resistência ao arrancamento de parafuso até a proporção de $10 \%$.

O fato do parafuso de topo estar fixado no centro do painel, onde geralmente ocorre a menor densidade, pode favorecer a resultados inferiores (CUNHA et al., 2014). Porém essa relação não foi evidenciada nos tratamentos pesquisados, não havendo diferenças estatísticas entre o ensaio de arrancamento de parafuso na superfície ou no topo. Situação semelhante foi referida por Semple; Smith (2006).

Painéis aglomerados em que foram adicionadas pequenas quantidades de tanino à ureia formaldeído, apesar de não ter proporcionado uma resistência ao arrancamento de parafuso igual àqueles em foram utilizados apenas ureia formaldeído, mostraram resistência superior ao mínimo estabelecido por instrumento normativo (CARNEIRO et al., 2009).

Tabela 5. Valores médios para o ensaio de resistência ao arrancamento de parafuso na superfície (APS) e no topo (APT).

Table 5. Average values of screw withdrawal on the surface (APS) and top (APT).

\begin{tabular}{|c|c|c|}
\hline \multirow[b]{2}{*}{ Adesivos utilizados } & \multicolumn{2}{|c|}{ Propriedade Mecânica } \\
\hline & $\begin{array}{c}\text { Arrancamento de parafuso na super- } \\
\text { fície } \\
\text { (APS) }\end{array}$ & $\begin{array}{c}\text { Arrancamento de parafuso no topo } \\
\text { (APT) }\end{array}$ \\
\hline \multirow{2}{*}{$100 \%$ UF } & $3,07 \mathrm{a}^{\#}$ & $2,82 \mathrm{a}$ \\
\hline & $(0,74)$ & $(0,70)$ \\
\hline \multirow{2}{*}{$100 \% \mathrm{TF}$} & $2,48 \mathrm{ab}$ & $1,93 \mathrm{ab}$ \\
\hline & $(0,84)$ & $(1,22)$ \\
\hline \multirow{2}{*}{$98 \% \mathrm{UF}+2 \% \mathrm{TF}$} & $1,57 \mathrm{~b}$ & $0,95 \mathrm{~b}$ \\
\hline & $(0,50)$ & $(0,34)$ \\
\hline \multirow{2}{*}{$95 \% \mathrm{UF}+5 \% \mathrm{TF}$} & $2,38 \mathrm{ab}$ & $1,68 \mathrm{ab}$ \\
\hline & $(0,55)$ & $(0,60)$ \\
\hline \multirow{2}{*}{$90 \% \mathrm{UF}+10 \% \mathrm{TF}$} & $3,03 \mathrm{a}$ & $2,37 \mathrm{a}$ \\
\hline & $(0,99)$ & $(1,65)$ \\
\hline \multirow{2}{*}{$85 \% \mathrm{UF}+152 \% \mathrm{TF}$} & $2,54 \mathrm{ab}$ & $1,78 \mathrm{ab}$ \\
\hline & $(0,59)$ & $(0,43)$ \\
\hline \multirow{2}{*}{ Média geral } & 2,51 & 1,92 \\
\hline & $(0,85)$ & $(1,07)$ \\
\hline
\end{tabular}

* UF: Ureia formaldeído, TF: Tanino formaldeído, \# Médias seguidas pela mesma letra, na coluna, não diferem estatisticamente entre si (Tukey <0,05). Valor entre parêntese é o desvio padrão. 


\section{Conclusões}

Com base nos resultados obtidos pode-se concluir que: - Apenas os testes de inchamento em espessura e ligação interna atingiram o valor mínimo estipulado pelo instrumento normativo.

- $\mathrm{O}$ adesivo químico alterado pelo tanino juntamente com as demais características do processo utilizado não é indicado, haja visto que, para os testes realizados, a qualidade dos painéis foi inferior aos do adesivo comercial ureia formaldeído puro.

- A falta de padronização da matéria-prima, como fator casca não controlável desencadeou resultados insatisfatórios.

- Sugere-se novos estudos com mistura de matéria-prima virgem, de menor densidade e, homogênea, juntamente à matéria-prima utilizada.

- Sugere-se novos estudos com aumento do teor de adesivos e mesmo tipo de partículas.

\section{Referências}

ANTWI-BOASIAKO, C.; ANIMAPAUH, S.O. Tannin extraction from the barks of three tropical hardwoods for the production of adhesives. Journal of Applied Sciences Research, v. 8, n. 6, p. 2959-2965, 2012.

ASSOCIAÇÃO BRASILEIRA DE NORMAS TÉCNICAS. NBR 11941: madeira - determinação da densidade básica. Rio de Janeiro, 2003.

ASSOCIAÇÃO BRASILEIRA DE NORMAS TÉCNICAS. NBR 14.810-3: chapas de madeira aglomerada - métodos de ensaios. Rio de Janeiro, 2006.

ASSOCIAÇÃO BRASILEIRA TÉCNICA DE CELULOSE E PAPEL. ABTCP M 11/77: Normas técnicas. São Paulo: ABTCP, 1974.

CARNEIRO, A.C.O.; VITAL, B.R.; FREDERICO, P.G.U.; CARVALHO, A.M.M.L.; VIDAURRE, G.B. Propriedades de chapas de aglomerado fabricadas com adesivo tânico de angico-vermelho (Anadenanthera peregrina) e uréia-formaldeído. Revista Árvore, Viçosa, v. 33, n. 3, p. 521-531, 2009.
CARNEIRO, A.C.O.; VITAL, B.R.; PIMENTA, A.S.; DELLA LUCIA, R.M. Propriedades de chapas de flocos fabricadas com adesivo de ureia formaldeído e de taninos de casca de Eucalyptus grandis W. Hill ex Maiden ou de Eucalyptus pellita F. Muell. Revista Árvore, Viçosa, v. 28, n. 5, p. 715-724, 2004.

CARVALHO, A.G.; LELIS, R.C.C; NASCIMENTO, AM. Avaliação de adesivos à base de taninos de Pinus caribaea var. bahamensis e de Acacia mearnsii na fabricação de painéis aglomerados. Ciência Florestal, Santa Maria, v. 24, n. 2, p. 479-489, 2014.

COMMERCIAL STANDARD. CS 236-66: Mat formed wood particleboard. Wallingford, 1968.

CUNHA, A.B.; LONGO, B.; RODRIGUES, A.A.; BREHMER, D.R. Produção de painéis de madeira aglomerada de Eucalyptus benthamii, Eucalyptus duinnii e Eucalyptus grandis. Scientia Forestalis, Piracicaba, v. 42, n. 102, p. 259-267, 2014.

DACOSTA, L.P.E.; HASELEIN, C.R.; SANTINI, E.J.; SCHNEIDER, P.R.; CALEGARI, L. Propriedades físicas de chapas de partículas aglomeradas fabricadas com resíduos do processamento mecânico da madeira de Pinus elliotii Engelm. Ciência Florestal, Santa Maria, v. 15, n. 4, p. 421-429, 2005.

GHALEHNO, M.D.; NAZERIASN, M. Physical and mechanical properties of particleboard from roselle (Hibiscus sabdariffa) stalks and eucalyptus (Eucalyptus camaldulensis) wood particles. Wood Material Science \& Engineering, Vantaa, v. 7, n. 1, p. 25-29, 2012.

ELBADAWI, M.; OSMAN, Z.; PARIDAH, T.; NASROUN, T.; KANTINER, W. Properties of particleboards made from Acacia seyal var. seyal using UF-tannin modified adhesives. Cellulose Chemistry and Technology, Romênia, v. 49, n. 34, p. 369-374, 2015.

FRIHART, C.R.; HUNT, C.G. Adhesives with wood materials bond formation and performance. In: Wood Handbook - Wood as an Engineering Material. Forest Products Laboratory. General Technical Report FPL-GTR-190. Chapter 10, Madison, WI: U.S. Department of Agriculture, Forest Service, Forest Products Laboratory. 2010.

GONÇALVES, F.G.; LELIS, R.C.C. Caracterização tecnológica da madeira de Acacia mangium Willd em plantio consorciado com eucalipto. Floresta e Ambiente, Seropédica, v. 19, n. 3, p. 286-295, 2012. 
GONÇALVES, F. G.; LELIS, R. C. C. Propriedades de duas resinas sintéticas após adição de tanino modificado. Floresta e Ambiente, Seropédica, v. 16, n. 2, p. 01-07, 2009.

GONÇALVES, F.G.; LELIS, R.C.C.; OLIVEIRA, J.T. Influência da composição da resina tanino-ureia-formaldeído nas propriedades físicas e mecânicas de chapas aglomeradas. Revista Árvore, Viçosa, v. 32, n. 04, p. 715-722, 2008.

HASELEIN, C.R.; CALEGARI, L.; BARROS, M.V.; HACK, C.; HILLIG, E.; PAULESKI, D.T.; POZZERA, F. Resistência mecânica e à umidade de painéis aglomerados com partículas de madeira de diferentes dimensões. Ciência Florestal, Santa Maria, v.12, n. 2, p. 127-134, 2002.

HILLING, E.; HASELEIN, C.R.; SANTINI, E.J. Propriedades mecânicas de chapas aglomeradas estruturais fabricadas com madeiras de pinus, eucalipto e acácia-negra. Revista Ciência Florestal, Santa Maria, v. 12, n. 1, p. 59-70, 2002.

IRLE, M.; BARBU, M.C. Wood-Based Panel Technology. In: Wood-Based Panels: An Introduction for Specialists, Thoemen, H.; Irle, M.; Sernek, M. (Eds), Chapter 1. Brunel University Press: London, 2010.

IWAKIRI, S. Painéis de Madeira Reconstituída. FUPEF, 2005.

IWAKIRI, S.; CUNHA, A.B; ALBUQUERQUE, C.E.C.; GORNIAK, E.; MENDES, L.M. Resíduos de serrarias na produção de painéis de madeira aglomerada de eucalipto. Scientia Agrária, Curitiba, v. 1, n. 1-2, p. 23-28, 2000.

MALONEY, T.M. Modern particleboard \& dry-process fiberboard manufacturing. Miller Freeman Inc., 1993.

MELO, R.R.; SANTINI, E.J.; HASELEIN, C.R.; STANGERLIN, D.M.; MÜLLER, M.T.; DEL MENEZZI, C.H.S. Avaliação das propriedades físico-mecânicas de painéis aglomerados de Eucalyptus grandis colados com ureia-formaldeído e tanino-formaldeído. Floresta, Curitiba, v. 40, n. 3, p. 497-506, 2010.

MELO, R.R.; DEL MENEZZI, C.H.S. Influência da massa específica nas propriedades físico-mecânicas de painéis aglomerados. Silva Lusitana, Lisboa, v. 18, p. 59-73, 2010.

MIYAZAKI, J.; HIRABAYASHI, Y. Effect of the addition of Acacia mangium bark on thermosetting of phenol-formaldehyde resin. Wood Science Technology, Berlim, v. 45, n. 3, p. 449-460, 2011.
MOSLEMI, A.A. Particleboard. Carbondele: Southern Illinois Press, v.1, 1974.

NEGRÃO, W.H.; SILVA, S.A.M.; CHRISTOFORO, A.L.; LAHR, F.A.R. Painéis aglomerados fabricados com mistura de partículas de madeiras tropicais. Ambiente Construído, Porto Alegre, v. 14, n. 3, p. 103-112, 2014.

OKINO, E.Y.A.; ANDAHUR, J.P.V.; SANTANA, M.A.E.; SOUZA, M.R. Resistência físico-mecânica de chapas aglomeradas de bagaço de cana-de-açúcar modificado quimicamente. Scientia Forestalis, Piracicaba, n. 52, p. 35-42, 1997.

PAN, Z.; ZHENG, Y.; ZHANG, R.; JENKINS, B.M. Physical properties of thin particleboard made from saline eucalyptus. Industrial Crops and Products, Amsterdam, v. 26, n. 2, p. 185-194, 2007.

PEDRAZZI, C.; HASELEIN, C.R.; SANTINI, E.J.; SCHNEIDER, P.R. Qualidade de chapas de partículas de madeira aglomerada fabricadas com resíduos de uma indústria de celulose. Ciência Florestal, Santa Maria, v. 16, n.2, p. 201-212, 2006.

PIZZI, A. Natural Phenolic Adhesives I: Tannin. In: PIZZI, A. \& MITTAL, K.L. (Ed.). Handbook of Adhesive Technology. $2^{\text {nd }}$ Edition. Marcel Dekker, 2003.

PIZZI, A. Urea-formaldehyde adhesives. In: PIZZI, A. \& MITTAL, K.L. (Ed.). Handbook of Adhesive Technology. Marcel Dekker, 1994.

ROWELL, R.M.; PETTERSEN, R.; HAN, J.S.; ROWELL, J.S.; TSHABALALA, M.A. Cell Wall Chemistry. In: Wood Chemistry and Wood Composites, Rowell, R. M. (Ed.), Chapter 3. Boca Raton: Florida, 2005.

SANCHES, F.L.; HILLIG, E.; IWAKIRI, S.; NAPOLI, L.M. Resistência de painéis aglomerados produzidos com mistura de madeira de espécies florestais tradicionais e não tradicionais. Ciência Florestal, Santa Maria, v. 26, n. 2, p. 559-569, 2016.

SEMPLE, K.E.; SMITH, G.D. Prediction of internal bond strength in particleboard from screw withdrawal resistance models. Wood and Fiber Science, Hanover, v. 38, n. 2, p. 256267, 2006.

STARK, N.M.; CAI, Z.; CARLL, C. Wood-Based Composite Materials: Panel Products, Glued-Laminated Timber, Structural Composite Lumber, and Wood-Nonwood Composite Materials. In: Wood Handbook - Wood as an engineering material. General Technical Report FPL-GTR-190. Madison, 
WI: U.S. Department of Agriculture, Forest Service, Forest Products Laboratory. 508 p., 2010.

TECHNICAL ASSOCIATION OF THE PULP AND PAPER INDUSTRY. TAPPI: T204 om-88: test methods - solvent extractives of wood and pulp. Atlanta: Tappi Press, 1998.

WEGNER, T.H.; BAKER, A.J.; BENDTSEN, B.A.; HIGHLEY, T.L.; HOWARD, J.L.; LEVAN, S.L.; MILLER, R.B.; MINOR, J.L.; PETTERSEN, R.C.; ROWELL, R.M.; SIMPSON, W.T.; YOUNGQUIST, J.A.; ZINKEL, D.F. Wood. In: MarkBikales-Overberger-Menges Encyclopedia of Polymer Science and Engineering. USDA, Forest Service Forest Products Laboratory, v. 17, $2^{\text {nd }}$ Ed., John Wiley \& Sons, 1989. 